\title{
REDUCTION OF GRADIENT RICCI SOLITON EQUATION
}

\author{
Benedito Leandro and João Paulo dos Santos \\ Universidade Federal de Goiás, Instituto de Matemática e Estatística \\ Goiânia, 74001-970, Brazil; bleandroneto@ufg.br \\ Universidade de Brasília, Departmento de Matemática \\ 70910-900, Brasília-DF, Brazil; joaopsantos@unb.br
}

\begin{abstract}
We consider gradient Ricci solitons conformal to an $n$-dimensional pseudo-Euclidean space and we describe entirely the most general substitution that reduces the resulting system of partial differential equations, originated from the gradient Ricci soliton equations, to a system of ordinary differential equations. As a consequence, the gradient Ricci solitons that arise from the reduced system are invariant under the action of either an $(n-1)$-dimensional translation group or the pseudo-orthogonal group acting on the corresponding $n$-dimensional pseudo-Euclidean space. The reduced system of ordinary differential equations is given. From such a system, particular solutions are obtained.
\end{abstract}

\section{Introduction and main results}

A pseudo-Riemannian manifold $\left(M^{n}, g\right)$ endowed with a smooth function $f$ is a gradient Ricci soliton if

$$
\operatorname{Ric}_{g}+\frac{1}{2} \mathcal{L}_{X} g=\lambda g
$$

where $\operatorname{Ric}_{g}$ is the Ricci tensor, $\mathcal{L}_{X} g$ is the Lie derivative in the direction of $X$ and $\lambda$ is a real constant. The vector field $X$ is called potential vector field. The Ricci soliton is called shrinking when $\lambda>0$, steady when $\lambda=0$, and expanding when $\lambda<0$. If $X$ is the gradient of a smooth function $f$, the Ricci soliton becomes

$$
\operatorname{Ric}_{g}+\operatorname{Hess}_{f}=\lambda g,
$$

where $\operatorname{Hess}_{f}$ is the Hessian of $f$. In this case, $\left(M^{n}, g\right)$ is called gradient Ricci soliton and the function $f$ is called potential function. Ricci solitons arise as self-similar solutions and they play an important role as singularity models for the Hamilton's Ricci flow.

Ricci solitons in pseudo-Riemannian manifolds have been recently investigated, as we can see in $[2,3,4,12]$, specially in the Lorentzian case. Explicit pseudoRiemannian conformally flat gradient steady Ricci solitons was obtained by Barbosa, Pina and Tenenblat in [1]. The authors firstly reduced a sytem of PDEs, that comes from the corresponding Ricci soliton equation, to a system of ODEs by considering a substitution by a function invariant under translations in a pseudo-Euclidean space. In the steady case, they provided all solutions of the reduced system.

https://doi.org/10.5186/aasfm.2020.4554

2010 Mathematics Subject Classification: Primary 53C21, 53C50, 53C44.

Key words: Gradient Ricci solitons, exact solutions, reduction, conformal metrics.

The second author was supported by FAPDF 0193.001346/2016. 
A substitution like the one considered by Barbosa et al. is known as an ansatz, i.e., a substitution that transforms a PDE into an ODE or a PDE with less independent variables. Using a rotational ansatz, Byrant showed the existence a complete, rotationally symmetric steady gradient Ricci soliton on $\mathbf{R}^{n}, n \geq 3$, which is unique up to homothety (see Chapter 1, Section 4 in [5]).

In this paper, we consider gradient Ricci solitons conformal to an $n$-dimensional pseudo-Euclidean space $\left(\mathbf{R}^{n}, g\right)$. We generalize the results obtained by Barbosa et al. by obtaining the most general ansatz that reduces the correspondent system of PDEs to a system of ODEs (see Theorem 1). It turns out that the function which plays the role of ansatz must be an invariant function under the action of the pseudo-orthogonal group or an invariant function under the group of translations. The reduced system of ODEs is given in Theorem 2. In Theorem 3, it is given a system of integro-differential equations equivalent to the reduced one. From such a system, particular solutions can easily be found it. These particular solutions are given in Corollaries 1 and 2.

The technique employed in the proof of Theorem 1 is a method used for finding ansätze for PDEs, known as the direct method of reduction, which was introduced in a systematic way by Clarkson and Kruskal [6], where the Boussinesq equation was considered. Although the direct method has been introduced for two-dimensional equations, it was also successful when multiple variables were considered, as we can see, for example, for the nonlinear multi-dimensional wave equations [9] and for the $n$-dimensional static vacuum Einstein equation [14]. The method consists in considering new variables given by unknown ansätze for a given PDE and then it is required that the equivalent system of equations be a system of ODEs or a system of PDEs with less independent variables. With this requirement, one obtains a set of partial differential equations for the ansätze. Once the ansätze are obtained, one has the reduced system.

In order to state our results, let us establish the notations and terminologies used in this paper, which are based on [13]. Let $\left(\mathbf{R}^{n}, g\right)$ be the standard pseudoEuclidean space with coordinates $\left(x_{1}, \ldots, x_{n}\right)$ and metric $g_{i j}=\delta_{i j} \varepsilon_{i}, 1 \leq i, j \leq n$, where $\varepsilon_{i}= \pm 1$. We want to find smooth functions $\varphi$ and $f$ defined on an open subset $\Omega \subset \mathbf{R}^{n}$ such that, for $\bar{g}$ given by

$$
\bar{g}=\frac{g}{\varphi^{2}}
$$

$(\Omega, \bar{g})$ is a gradient Ricci soliton with potential function $f$, i.e.,

$$
\operatorname{Ric}_{\bar{g}}+\operatorname{Hess}_{\bar{g}}(f)=\lambda \bar{g}
$$

where $\operatorname{Ric}_{\bar{g}}$ and $\operatorname{Hess}_{\bar{g}}(f)$ stand for the Ricci tensor and the Hessian of the metric $\bar{g}$, respectively. In what follows, we will denote the first and second partial derivatives of a function $F: \Omega \subset \mathbf{R}^{n} \rightarrow \mathbf{R}$ by

$$
F_{, i}:=\frac{\partial F}{\partial x_{i}} \quad \text { and } \quad F_{, i j}:=\frac{\partial^{2} F}{\partial x_{i} x_{j}}
$$

respectively. In this setting, Theorem 1.3 in [1] provides the correspondent system of PDEs for equation (1.3), i.e., $(\Omega, \bar{g})$ is a gradient Ricci soliton with potential function $f$ if and only if

$$
(n-2) \varphi_{, i j}+\varphi f_{, i j}+\varphi_{, i} f_{, j}+\varphi_{, j} f_{, i}=0, \quad i \neq j
$$


and for each $i$

$$
\varphi\left[(n-2) \varphi_{, i i}+\varphi f_{, i i}+2 \varphi_{, i} f_{, i}\right]+\varepsilon_{i} \sum_{k} \varepsilon_{k}\left[\varphi \varphi_{, k k}-(n-1) \varphi_{, k}^{2}-\varphi \varphi_{, k} f_{, k}\right]=\varepsilon_{i} \lambda
$$

It is important to note that, although Theorem 1.3 in [1] requires $n \geq 3$, it is not necessary to assume any restriction on the dimension.

Our main goal is to find a smooth function $\xi: \Omega \subset \mathbf{R}^{n} \rightarrow \mathbf{R}$ that will be an ansatz such that equations (1.4) and (1.5) are reduced to ordinary differential equations. Specifically, we want to find $\xi: \Omega \subset \mathbf{R}^{n} \rightarrow \mathbf{R}$ such that $f \circ \xi$ and $\varphi \circ \xi$ are solutions for such equations. The next theorem shows the most general form for the function $\xi$.

Theorem 1. Let $\left(\mathbf{R}^{n}, g\right)$ be the standard pseudo-Euclidean space with coordinates $\left(x_{1}, \ldots, x_{n}\right)$ and metric $g_{i j}=\delta_{i j} \varepsilon_{i}, 1 \leq i, j \leq n$, where $\varepsilon_{i}= \pm 1$. Then, there exists a smooth real-valued function $\xi$ on an open subset $\Omega \subset \mathbf{R}^{n}$ such the system of PDEs given by equations (1.4) and (1.5) are reduced to an system of ODEs with an independent variable $\xi$ if and only if

$$
\xi\left(x_{1}, \ldots, x_{n}\right)=(\Psi \circ P)\left(x_{1}, \ldots, x_{n}\right),
$$

where $P\left(x_{1}, \ldots, x_{n}\right)=\sum_{k=1}^{n}\left(\tau \varepsilon_{k} x_{k}^{2}+\alpha_{k} x_{k}+\beta_{k}\right), \tau, \alpha_{k}, \beta_{k} \in \mathbf{R}$ and $\Psi$ is a smooth real-valued function.

We observe that Theorem 1 is sharp in the following sense: there is no other function $\xi$, that depends on all variables $\left(x_{1}, \ldots, x_{n}\right)$, such that equations $(1.4)$ and (1.5) reduce to a system of ODEs. Let us also observe that the level functions of such a $\xi$ provides a foliation of the correspondent gradient Ricci soliton by hypersurfaces invariant under the action of the pseudo-orthogonal group, up to change of coordinates, when $\tau \neq 0$, or of the group of translations, when $\tau=0$, this latter is precisely the case considered in [1]. Fernández-López and García-Río [8], using the local decomposition of a Ricci soliton metric into a warped product metric proved that a locally conformally flat gradient Ricci soliton is rotationally symmetric. However, for an expanding soliton, they require that the curvature operator must be nonnegative, cf. [8, Remark 1]. From Theorem 1, we prove without any assumption on the curvature that a pseudo-Riemannian conformally flat gradient expanding (shrinking or steady) Ricci soliton, foliated by $n-1$ dimensional subsets invariant under isometries, is pseudo-rotationally symmetric (up to change of variables). Moreover, our proof is quite different in the sense that we do not use the warped product structure.

Once we have the most general ansatz, one has the reduced system of ODEs. From (1.6) we can consider $\varphi$ and $f$ smooth real-valued functions depending on the variable $\xi=\sum_{k}\left(\tau \varepsilon_{k} x_{k}^{2}+\alpha_{k} x_{k}+\beta_{k}\right)$. In this case, let us write

$$
\varphi^{\prime}:=\frac{d \varphi}{d \xi} \quad \text { and } \quad f^{\prime}:=\frac{d f}{d \xi}
$$

and then the reduced system is given by the following theorem

Theorem 2. Let $\left(\mathbf{R}^{n}, g\right)$ be a pseudo-Euclidean space with coordinates $\left(x_{1}, \ldots, x_{n}\right)$ and metric $g_{i j}=\delta_{i j} \varepsilon_{i}, 1 \leq i, j \leq n$, where $\varepsilon_{i}= \pm 1$. Let $\Omega \subset \mathbf{R}^{n}$ be an open subset and consider smooth functions $\varphi(\xi)$ and $f(\xi)$ such that $\xi: \Omega \subset \mathbf{R}^{n} \rightarrow \mathbf{R}$ is given by $\xi=\sum_{k}\left(\tau \varepsilon_{k} x_{k}^{2}+\alpha_{k} x_{k}+\beta_{k}\right)$. Then $(\Omega, \bar{g})$ is a gradient Ricci soliton with potential function $f(\xi)$ and $\bar{g}=g /(\varphi(\xi))^{2}$ if and only if the functions $\varphi$ and $f$ satisfy

$$
(n-2) \varphi^{\prime \prime}+f^{\prime \prime} \varphi+2 \varphi^{\prime} f^{\prime}=0
$$


and

$$
2 \tau \varphi\left[2(n-1) \varphi^{\prime}+\varphi f^{\prime}\right]+\left[\varphi \varphi^{\prime \prime}-(n-1)\left(\varphi^{\prime}\right)^{2}-\varphi \varphi^{\prime} f^{\prime}\right](4 \tau \xi+\Lambda)=\lambda,
$$

where $\tau, \alpha_{k}, \beta_{k} \in \mathbf{R}$ and $\Lambda=\sum_{k}\left(\varepsilon_{k} \alpha_{k}^{2}-4 \tau \beta_{k}\right)$.

An example of complete solution for Theorem 2 is the Gaussian soliton. In fact, it is enough to consider $\varphi=k$, where $k$ is constant, and $\tau \neq 0$. Therefore, from (1.7) we have that $f(\xi)=a_{1} \xi+a_{2}$, where $a_{1}, a_{2} \in \mathbf{R}$. From (1.8) we can see that $a_{1}=\frac{\lambda}{2 \tau k^{2}}$. Considering $\alpha_{i}=\beta_{i}=0$ for all $i \in\{1, \ldots, n\}$ in the expression of $\xi$, we have the Gaussian soliton. On the other hand, when $f$ is constant, it follows from (1.7) that $\varphi(\xi)=b_{1} \xi+b_{2}$, which implies $\bar{g}$ has constant curvature. Consequently, the space forms are complete solutions of Theorem 2 .

When $\tau=0$ and $n \geq 3$, we recover the ODEs given in [1]. In this case, if we consider $\lambda=0$ we have the explicit solutions given in [1].

Note that equation (1.7) determines $f^{\prime}$ by means of $\varphi$. Consequently, we can write (1.8) completely in terms of $\varphi$. If we write $\phi=\log \varphi^{2}$, we have the following equivalent system of integro-differential equations:

Theorem 3. Let $\left(\mathbf{R}^{n}, g\right)$ be a pseudo-Euclidean space with coordinates $\left(x_{1}, \ldots\right.$, $x_{n}$ ) and metric $g_{i j}=\delta_{i j} \varepsilon_{i}, 1 \leq i, j \leq n$, where $\varepsilon_{i}= \pm 1$. Let $\Omega \subset \mathbf{R}^{n}$ be an open subset and consider smooth functions $\phi(\xi)$ and $f(\xi)$ such that $\xi: \Omega \subset \mathbf{R}^{n} \rightarrow \mathbf{R}$ is given by $\xi=\sum_{k}\left(\tau \varepsilon_{k} x_{k}^{2}+\alpha_{k} x_{k}+\beta_{k}\right)$. Then $(\Omega, \bar{g})$ is a gradient Ricci soliton with potential function $f(\xi)$ and $\bar{g}=e^{-\phi} g$ if and only if the functions $\phi$ and $f$ satisfy

$$
\begin{aligned}
f^{\prime}-\mathcal{J}(\phi) e^{-\phi}+\frac{n-2}{2} \phi^{\prime} & =0 \\
T \phi^{\prime \prime}+2 n \tau \phi^{\prime}+\mathcal{J}(\phi)\left(T e^{-\phi}\right)^{\prime}-2 \lambda e^{-\phi} & =0 .
\end{aligned}
$$

for$$
\mathcal{J}(\phi)=c_{1}+\frac{n-2}{4} \int e^{\phi}\left(\phi^{\prime}\right)^{2} d \xi,
$$$$
T=4 \tau \xi+\Lambda,
$$

where $c_{1}, \tau, \alpha_{k}, \beta_{k} \in \mathbf{R}$ and $\Lambda=\sum_{k}\left(\varepsilon_{k} \alpha_{k}^{2}-4 \tau \beta_{k}\right)$.

Particular solutions given by Theorem 3 are described in the following two examples:

Example 1. The equations in Theorem 3 have a simple form when $n=2$. Recall that any Riemannian or Lorentzian 2-dimensional manifold is locally conformally flat. Consequently, when we consider $n=2$ in Theorem 3 , we have the differential equations that describe all gradient Ricci solitons in 2-dimensional pseudo-Riemannian or Riemannian manifolds. In this case, the functions $\phi$ and $f$ satisfy

$$
f^{\prime}=c_{1} e^{-\phi}
$$

and

$$
\left[\left(\phi^{\prime}+c_{1} e^{-\phi}\right)(4 \tau \xi+\Lambda)\right]^{\prime}=2 \lambda e^{-\phi}
$$


where $\tau, \alpha_{k}, \beta_{k} \in \mathbf{R}$ and $\Lambda=\sum_{k}\left(\varepsilon_{k} \alpha_{k}^{2}-4 \tau \beta_{k}\right)$. In particular, for the steady case, the solutions of (1.14) are given by

$$
\begin{aligned}
e^{\phi} & =-\frac{c_{1}}{4 \tau-c_{2}}(4 \tau \xi+\Lambda)+c_{3}(4 \tau \xi+\Lambda)^{c_{2} / 4 \tau}, & & \text { if } c_{2} \neq 4 \tau, \\
e^{\phi} & =-\frac{c_{1}}{4 \tau}(4 \tau \xi+\Lambda) \log (4 \tau \xi+\Lambda)+c_{3}(4 \tau \xi+\Lambda), & & \text { if } c_{2}=4 \tau,
\end{aligned}
$$

for real constants $c_{2}$ and $c_{3}$. In fact, equation (1.14) becomes the following linear ODE for the function $Y=e^{\phi}$

$$
Y^{\prime}-\frac{c_{2} Y}{4 \tau \xi+\Lambda}=-c_{1}
$$

The solutions of $\phi$ are given by (1.15).

In the Riemannian case, we can choose $c_{1}=-1, c_{2}=0, c_{3}=1, \alpha_{i}=\beta_{i}=0$ and $\tau=1$ to obtain the cigar soliton $\left(\mathbf{R}^{2}, \bar{g}, f\right)$, where

$$
\bar{g}=\frac{d x_{1}^{2}+d x_{2}^{2}}{1+x_{1}^{2}+x_{2}^{2}} \quad \text { and } \quad f\left(x_{1}, x_{2}\right)=-\log \left(1+x_{1}^{2}+x_{2}^{2}\right)
$$

It is important to observe that the case $n=2$ was not considered in [1]. Therefore, when $\tau=0$, Example 1 complements the results contained therein.

Example 2. A second class of solutions provided by Theorem 3 is when $e^{\phi}=$ $T^{2 m}$, for $T(\xi)=4 \tau \xi+\Lambda, \tau \neq 0$, and a real constant $m$. Since $m=0$ and $m=1$ provide trivial Ricci solitons, in what follows we will consider $m \neq 0$ and $m \neq 1$. In this case, we have:

$$
\mathcal{J}(\phi)= \begin{cases}c_{1}+4(n-2) m^{2} \tau c_{2}+\frac{4 \tau m^{2}(n-2)}{2 m-1} T^{2 m-1}, & m \neq \frac{1}{2}, \\ c_{1}+(n-2) \tau c_{2}+(n-2) \tau \log (T), & m=\frac{1}{2}\end{cases}
$$

If $m \neq \frac{1}{2}$, equation (1.10) becomes the following polynomial equation on $T$ :

$$
2 \tau(1-2 m)\left(c_{1}+4(n-2) m^{2} \tau c_{2}\right)-\lambda+8 \tau^{2} m(1-m)(n-2) T^{2 m-1}=0 .
$$

Therefore, the only possibility one has is $n=2$ and $2 \tau(1-2 m) c_{1}=\lambda$. In this case, the corresponding non-trivial gradient Ricci soliton is shrinking or expanding, with potential function given by

$$
f(\xi)=\frac{c_{1}}{4 \tau(1-2 m)}(4 \tau \xi+\Lambda)^{1-2 m}+c_{2} .
$$

When $m=\frac{1}{2}$, a straightforward computation shows that (1.10) reduces to $4(n-2) \tau^{2}=\lambda$. In this case, the corresponding non-trivial gradient Ricci soliton is shrinking or steady, with potential function given by

$$
f(\xi)=\frac{2 c_{1}+\left(c_{2}-2\right) \tau(n-2)}{8 \tau} \log (4 \tau \xi+\Lambda)+\frac{n-2}{8}[\log (4 \tau \xi+\Lambda)]^{2}+c_{3} .
$$

Remark 1. In the pseudo-Riemannian setting, the causal character of $\nabla f$ is given by the sign of $4 \tau \xi+\Lambda$, since $|\nabla f|^{2}=\left(\varphi f^{\prime}\right)^{2}(4 \tau \xi+\Lambda)$. In our examples, the isotropic case is only allowed in Example 1, where we can obtain infinitely many steady gradient Ricci solitons since equation (1.14) is trivially satisfied. Example 2 provides only solutions where $\nabla f$ is non-isotropic, since $\tau \neq 0$ in this case. The causal character of $\nabla f$ may change accordingly to the constants involved. 
Remark 2. Examples 1 and 2 are given explicitly with well-determined constants $\lambda$ and they are unique up to initial conditions. In Example 1, we consider the steady case, while Example 2 provides shrinking, steady or expanding gradient Ricci solitons, depending on a relation of constants (given by the initial conditions, the dimension and the type of the solutions) that determines $\lambda$. Since two Ricci soliton potential vector fields differ in a homothetic vector field (see [2]), Examples 1 and 2 may be considered to explicitly obtain new examples, by adding homothetic vector fields to the potential vector fields obtained there.

\section{Proofs of the main results}

This section is reserved for the proofs of the main results of this paper. Let us start with the following lemma:

Lemma 1. Let $\left(\mathbf{R}^{n}, g\right)$ be a pseudo-Euclidean space with cartesian coordinates $\left(x_{1}, \ldots, x_{n}\right)$ and metric $g_{i j}=\delta_{i j} \varepsilon_{i}, 1 \leq i, j \leq n$, where $\varepsilon_{i}= \pm 1$ which at least one equal to 1 . Let $\Omega \subset \mathbf{R}^{n}$ be an open subset and $f, \varphi: \Omega \rightarrow \mathbf{R}$ be smooth functions. If $(\Omega, \bar{g})$ is a gradient Ricci soliton with potential function $f$ and $\bar{g}=g / \varphi^{2}$, then the functions $\varphi$ and $f$ satisfy

$$
n \varepsilon_{i}\left[(n-2) \varphi_{, i i}+\varphi f_{, i i}+2 \varphi_{, i} f_{, i}\right]=\sum_{k} \varepsilon_{k}\left[(n-2) \varphi_{, k k}+\varphi f_{, k k}+2 \varphi_{, k} f_{, k}\right] .
$$

Proof. Taking the trace of equation (1.3) we obtain

$$
R_{\bar{g}}+\Delta_{\bar{g}} f=n \lambda .
$$

It is well known that if $\bar{g}=g / \varphi^{2}$ (cf. Lemma 1 in [10]), then

$$
\operatorname{Ric}_{\bar{g}}=\frac{1}{\varphi^{2}}\left\{(n-2) \varphi \operatorname{Hess}_{g}(\varphi)+\left[\varphi \Delta_{g} \varphi-(n-1)\left|\nabla_{g} \varphi\right|^{2}\right] g\right\} .
$$

Hence, the scalar curvature of $\bar{g}$ is given by

$$
\begin{aligned}
R_{\bar{g}} & =\sum_{k=1}^{n} \varepsilon_{k} \varphi^{2}\left(\operatorname{Ric}_{\bar{g}}\right)_{k k}=(n-1)\left(2 \varphi \Delta_{g} \varphi-n\left|\nabla_{g} \varphi\right|^{2}\right) \\
& =(n-1)\left[2 \varphi \sum_{k} \varepsilon_{k} \varphi_{, k k}-n \sum_{k} \varepsilon_{k}\left(\varphi_{, k}\right)^{2}\right] \\
& =\sum_{k} \varepsilon_{k}\left[2(n-1) \varphi \varphi_{, k k}-n(n-1)\left(\varphi_{, k}\right)^{2}\right]
\end{aligned}
$$

On the other hand,

$$
\Delta_{\bar{g}} f=\sum_{k} \varepsilon_{k}\left[\varphi^{2} f_{, k k}-(n-2) \varphi \varphi_{, k} f_{, k}\right] .
$$

Then, from (2.2), (2.3) and (2.4) we have

$$
\sum_{k} \varepsilon_{k}\left[2(n-1) \varphi \varphi_{, k k}-n(n-1) \varphi_{, k}^{2}+\varphi^{2} f_{, k k}-(n-2) \varphi \varphi_{, k} f_{, k}\right]=n \lambda .
$$

Multiplying (1.5) by $n \varepsilon_{i}$ and comparing with (2.5) we obtain the (2.1).

Proof of Theorem 1. By hypothesis $f(\xi)$ and $\varphi(\xi)$ are functions of $\xi$. Then, from (1.4) we have the following equation

$$
(n-2)\left[\varphi^{\prime \prime} \xi_{, i} \xi_{, j}+\varphi^{\prime} \xi_{, i j}\right]+\varphi\left[f^{\prime \prime} \xi_{, i} \xi_{, j}+f^{\prime} \xi_{, i j}\right]+2 \varphi^{\prime} f^{\prime} \xi_{, i} \xi_{, j}=0,
$$


which is rewritten as

$$
\left[(n-2) \varphi^{\prime \prime}+\varphi f^{\prime \prime}+2 \varphi^{\prime} f^{\prime}\right] \xi_{, i} \xi_{, j}+\left[(n-2) \varphi^{\prime}+\varphi f^{\prime}\right] \xi_{, i j}=0
$$

Dividing (2.6) by $\xi_{, i} \xi_{, j}$ we conclude that

$$
F(\xi)=\frac{\xi_{, i j}}{\xi_{, i} \xi_{, j}}
$$

for some smooth function $F(\xi)$. On the other hand, equation (2.1) in Lemma 1 implies that

$\left[(n-2) \varphi^{\prime \prime}+\varphi f^{\prime \prime}+2 \varphi^{\prime} f^{\prime}\right]\left[n \varepsilon_{i} \xi_{, i}^{2}-\sum_{k} \varepsilon_{k} \xi_{, k}^{2}\right]+\left[(n-2) \varphi^{\prime}+\varphi f^{\prime}\right]\left[n \varepsilon_{i} \xi_{, i i}-\sum_{k} \varepsilon_{k} \xi_{, k k}\right]=0$.

From (2.6), (2.7) and the above equation we have

$$
\left[(n-2) \varphi^{\prime}+\varphi f^{\prime}\right]\left\{\left[n \varepsilon_{i} \xi_{, i i}-\sum_{k} \varepsilon_{k} \xi_{, k k}\right]-F(\xi)\left[n \varepsilon_{i} \xi_{, i}^{2}-\sum_{k} \varepsilon_{k} \xi_{, k}^{2}\right]\right\}=0 .
$$

Observe that the soliton is trivial if $(n-2) \varphi^{\prime}+\varphi f^{\prime}=0$. In fact, supposing that, the first derivative of this equation leads us to

$$
(n-2) \varphi^{\prime \prime}+\varphi f^{\prime \prime}+\varphi^{\prime} f^{\prime}=0 .
$$

Therefore, from (2.6) we have $\varphi^{\prime} f^{\prime} \xi_{, i} \xi_{, j}=0$. This equation implies that either $\varphi$ or $f$ are constant functions. In any case, we have that $\varphi$ and $f$ must be trivial. Consequently, from (2.8) we conclude that

$$
\left[n \varepsilon_{i} \xi_{, i i}-\sum_{k} \varepsilon_{k} \xi_{, k k}\right]=F(\xi)\left[n \varepsilon_{i} \xi_{, i}^{2}-\sum_{k} \varepsilon_{k} \xi_{, k}^{2}\right]
$$

and, consequently,

$$
n \varepsilon_{i}\left[\left(\xi_{, i}\right) e^{-\int F d \xi}\right]_{, i}=\sum_{k} \varepsilon_{k}\left[\left(\xi_{, k}\right) e^{-\int F d \xi}\right]_{, k} .
$$

At this point, let us observe that equation (2.7) can be integrated as the following

$$
\log \left(\xi_{, i}\right)=\int F d \xi+F_{i}\left(\hat{x}_{j}\right)
$$

where the symbol $\hat{x}_{j}$ denotes that $F_{i}$ does not depend on the variable $x_{j}$. By considering this process for all $j \neq i$, we have

$$
\xi_{, i}=e^{\int F d \xi+F_{i}\left(x_{i}\right)}=G_{i} G,
$$

where $G_{i}\left(x_{i}\right)=e^{F_{i}\left(x_{i}\right)}$ and $G(\xi)=e^{\int F d \xi}$. Therefore, equations (2.9) and (2.10) imply that

$$
n \varepsilon_{i} G_{i, i}=\sum_{k} \varepsilon_{k} G_{k, k} .
$$

Observe that the left side of the above equation depends only on the variable $x_{i}$, thus this implies that $G_{i}\left(x_{i}\right)=2 \tau \varepsilon_{i} x_{i}+\alpha_{i}$, where $\alpha_{i}$ and $\tau$ are real constants. Since $\frac{\xi_{, i}}{G_{i}}=G$ we have for all $i \neq j$ that

$$
\frac{\xi_{, i}}{2 \tau \varepsilon_{i} x_{i}+\alpha_{i}}=\frac{\xi_{, j}}{2 \tau \varepsilon_{j} x_{j}+\alpha_{j}} .
$$


The characteristic for equation (2.11) implies that

$$
\xi=\Psi\left(\sum_{i} \tau \varepsilon_{i} x_{i}^{2}+\alpha_{i} x_{i}+\beta_{i}\right)
$$

where $\Psi$ is a smooth function. Conversely, let us suppose that $\xi=\Psi\left(\sum_{i} \tau \varepsilon_{i} x_{i}^{2}+\right.$ $\alpha_{i} x_{i}+\beta_{i}$ ), for a real smooth function $\Psi$ and constants $\tau, \alpha_{i}, \beta_{i}$. Since we are considering smooth functions by $f(\xi)$ and $\varphi(\xi)$ as solutions of (1.4) and (1.5), we can suppose, without loss of generality, that $\xi=\sum_{k} U_{k}\left(x_{k}\right)$, where $U_{k}\left(x_{k}\right)=\tau \varepsilon_{k} x_{k}^{2}+\alpha_{k} x_{k}+\beta_{k}$, then we have

$$
\begin{aligned}
& \varphi_{, i}=\varphi^{\prime} U_{i}^{\prime}, \quad \varphi_{, i j}=\varphi^{\prime \prime} U_{i}^{\prime} U_{j}^{\prime}, \quad \varphi_{, i i}=\varphi^{\prime \prime}\left(U_{i}^{\prime}\right)^{2}+\varphi^{\prime} U_{i}^{\prime \prime}, \\
& f_{, i}=f^{\prime} U_{i}^{\prime}, \quad f_{, i j}=f^{\prime \prime} U_{i}^{\prime} U_{j}^{\prime} \quad \text { and } \quad f_{, i i}=f^{\prime \prime}\left(U_{i}^{\prime}\right)^{2}+f^{\prime} U_{i}^{\prime \prime} .
\end{aligned}
$$

Hence, from equations (1.4) and (1.5) we get

$$
\left[(n-2) \varphi^{\prime \prime}+f^{\prime \prime} \varphi+2 \varphi^{\prime} f^{\prime}\right] U_{i}^{\prime} U_{j}^{\prime}=0
$$

and for $i=j$

$$
\begin{aligned}
& \varphi\left[(n-2) \varphi^{\prime \prime}+f^{\prime \prime} \varphi+2 \varphi^{\prime} f^{\prime}\right]\left(U_{i}^{\prime}\right)^{2}+\varphi\left[(n-2) \varphi^{\prime}+\varphi f^{\prime}\right] U_{i}^{\prime \prime} \\
& +\varepsilon_{i}\left\{\left[\varphi \varphi^{\prime \prime}-(n-1)\left(\varphi^{\prime}\right)^{2}-\varphi \varphi^{\prime} f^{\prime}\right] \sum_{k} \varepsilon_{k}\left(U_{k}^{\prime}\right)^{2}+\varphi \varphi^{\prime} \sum_{k} \varepsilon_{k} U_{k}^{\prime \prime}\right\}=\varepsilon_{i} \lambda
\end{aligned}
$$

From (2.13) we get

$$
(n-2) \varphi^{\prime \prime}+f^{\prime \prime} \varphi+2 \varphi^{\prime} f^{\prime}=0 .
$$

For the second equation we first have $\sum_{k} \varepsilon_{k}\left(U_{k}^{\prime}\right)^{2}=4 \tau \xi+\Lambda$ and $\sum_{k} \varepsilon_{k} U_{k}^{\prime \prime}=2 n \tau$, with $\Lambda=\sum_{k}\left(\varepsilon_{k} \alpha_{k}-4 \tau \beta_{k}\right)$. Using (2.15) we can write (2.14) as

$$
2 \tau \varphi\left[2(n-1) \varphi^{\prime}+\varphi f^{\prime}\right]+\left[\varphi \varphi^{\prime \prime}-(n-1)\left(\varphi^{\prime}\right)^{2}-\varphi \varphi^{\prime} f^{\prime}\right](4 \tau \xi+\Lambda)=\lambda .
$$

Proof of Theorem 2. Theorem 2 is a consequence of Theorem 1. The system of ODEs is given by equations (2.15) and (2.16).

Proof of Theorem 3. Since $\phi=\log \varphi^{2}$ and

$$
\frac{\varphi^{\prime \prime}}{\varphi}=\left(\frac{\varphi^{\prime}}{\varphi}\right)^{2}+\left(\frac{\varphi^{\prime}}{\varphi}\right)^{\prime}
$$

the equation (1.7) is equivalent to

$$
f^{\prime \prime}+\phi^{\prime} f^{\prime}+\frac{n-2}{2}\left(\frac{\left(\phi^{\prime}\right)^{2}}{2}+\phi^{\prime \prime}\right)=0
$$

The equation above is a first order linear ODE for the $f^{\prime}$ whose solution is given by (1.9). On the other hand, multiplying (1.8) by $2 \varphi^{-2}$ we have

$$
T^{\prime}\left(2(n-1) \frac{\varphi^{\prime}}{\varphi}+f^{\prime}\right)+2\left(\frac{\varphi^{\prime \prime}}{\varphi}-(n-1)\left(\frac{\varphi^{\prime}}{\varphi}\right)^{2}-\frac{\varphi^{\prime}}{\varphi} f^{\prime}\right) T=2 \lambda \varphi^{-2}
$$

where $T 4 \tau \xi+\Lambda$. Therefore, equations (2.17) and (2.18) imply

$$
\left(2 \frac{\varphi^{\prime}}{\varphi}\right)^{\prime} T+f^{\prime}\left(T^{\prime}-2 \frac{\varphi^{\prime}}{\varphi} T\right)+2 \frac{\varphi^{\prime}}{\varphi}\left((n-1) T^{\prime}+(2-n) \frac{\varphi^{\prime}}{\varphi} T\right)=2 \lambda \varphi^{-2} .
$$


By (1.9) and $\phi=\log \varphi^{2}$ we rewrite (2.19) as

$$
\begin{aligned}
& \phi^{\prime \prime} T+\left(\mathcal{J}(\phi) e^{-\phi}-\frac{(n-2)}{2} \phi^{\prime}\right)\left(T^{\prime}-\phi^{\prime} T\right) \\
& +\phi^{\prime}\left((n-1) T^{\prime}+\frac{(2-n)}{2} \phi^{\prime} T\right)=2 \lambda e^{-\phi} .
\end{aligned}
$$

Since $\left(T e^{-\phi}\right)^{\prime}=e^{-\phi}\left(T^{\prime}-\phi^{\prime} T\right)$, we have (1.10) from (2.20).

Acknowledgement. The authors are grateful to P. Bonfim, R. Pina, K. Tenenblat and the referee for the valuable suggestions on the elaboration of this manuscript.

\section{References}

[1] Barbosa, E., R. Pina, and K. Tenenblat: On gradient Ricci solitons conformal to a pseudo-Euclidean space. - Israel J. Math. 200, 2014, 213-224.

[2] Batat, W., M. Brozos-Vázquez, E. García-Río, and S. Gavino-Fernández: Ricci solitons on Lorentzian manifolds with large isometry groups. - Bull. Lond. Math. Soc. 43:6, 2011, 1219-1227.

[3] Brozos-Vázquez, M., G. Calvaruso, E. García-Río, and S. Gavino-Fernández: Threedimensional Lorentzian homogeneous Ricci solitons. - Israel J. Math. 188, 2012, 385-403.

[4] Brozos-Vázquez, M., E. García-Río, and S. Gavino-Fernández: Locally conformally flat Lorentzian gradient Ricci solitons. - J. Geom. Anal. 23:3, 2013, 1196-1212.

[5] Chow, B., S.-C. Chu, D. Glickenstein, C. Guenther, J. Isenberg, T. Ivey, D. Knopf, P. Lu, F. Luo, and L. NI: The Ricci flow: techniques and applications. Part I. Geometric aspects. - Math. Surveys Monogr. 135, Amer. Math. Soc., Providence, RI, 2007.

[6] Clarkson, P. A., and M. D. Kruskal: New similarity reductions of the Boussinesq equation. - J. Math. Phys. 30:10, 1989, 2201-2213.

[7] Fernández-López, M., and E. García-Río: Rigidity of shrinking Ricci solitons. - Math. Z. 269, 2011, 461-466.

[8] Fernández-López, M., and E. García-Río: A note on locally conformally flat gradient Ricci solitons. - Geom. Dedicata 168:1, 2014, 1-7.

[9] Fushchich, W. I., R. Z. Zhdanov, and I. A. Yegorchenko: On the reduction of the nonlinear multi-dimensional wave equations and compatibility of the D'Alembert-Hamilton system. - J. Math. Anal. Appl. 161:2, 1991, 352-360.

[10] Khunel, W.: Conformal transformations between Einstein spaces. - In: Conformal Geometry, ed. by R. S. Kulkarni and U. Pinkall, Aspects Math. E, vol. 12, Vieweg, Braunschweig, 1988, $105-146$.

[11] Kotschwar, B.: On rotationally invariant shrinking Ricci solitons. - Pacific J. Math. 236:1, 2008, 73-88.

[12] Onda, K.: Lorentz Ricci solitons on 3-dimensional Lie groups. - Geom. Dedicata 147, 2010, $313-322$.

[13] Pina, R., and K. Tenenblat: On solutions of the Ricci curvature equation and the Einstein equation. - Israel J. Math. 171, 2009, 61-76.

[14] Dos Santos, J. P., and B. Leandro: Reduction of the $n$-dimensional static vacuum Einstein equation and generalized Schwarzschild solutions. - J. Math. Anal. Appl. 469:2, 2019, 882-896. 